\title{
CHEMICAL AND SPECTROPHOTOMETRIC EVOLUTION OF OPTICALLY IDENTIFIED QSO ABSORPTION SYSTEMS
}

\author{
U. LINDNER, U. FRITZE-V. ALVENSLEBEN, C.S. MÖLLER AND \\ K.J. FRICKE \\ Universitäts-Sternwarte, Göttingen, Germany
}

We have compiled all published data on optically identified QSO absorption line systems (cf. Lindner, etal., 1996, A\&A 316, 123). This selection with respect to gas absorption cross section supplies us with essentially unbiased galaxy samples.

Our unified chemical and spectrophotometric evolutionary synthesis code has been extended to include stellar evolutionary tracks and element yields for 5 different metallicities. This allows to follow the spectrophotometric and chemical evolution of galaxies of various Hubble types in a chemically consistent way.

Comparison of observed apparent magnitudes and colors with model calculations reveals satisfactory agreement over much of the Hubble time between our galaxy evolution models and observational data and gives information about the spectral types of the absorbing galaxies. Data in additional passbands are necessary for constraints on the cosmological parameters. Model predictions serve to facilitate future identifications.

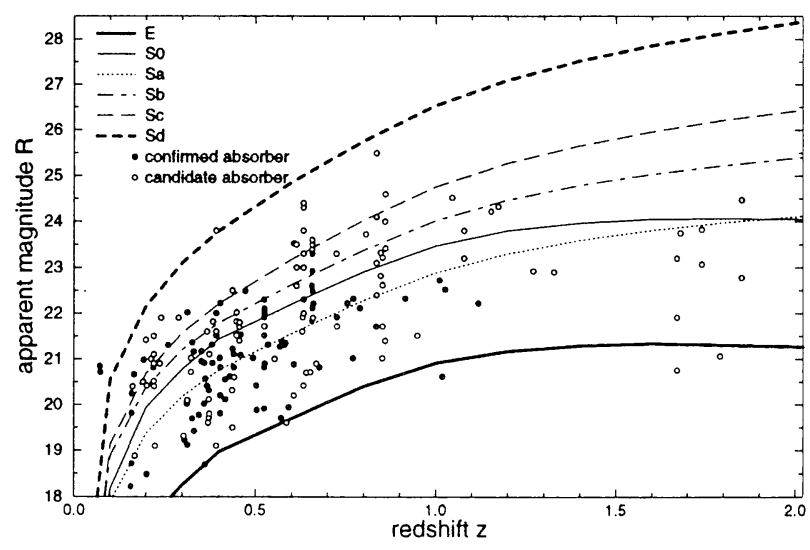

